\title{
Bispecific anti-CD3 $x$ anti-HER2 antibody mediates T cell cytolytic activity to HER2-positive colorectal cancer in vitro and in vivo
}

\author{
HUAMIN HAN ${ }^{1,2^{*}}$, JUAN MA $^{1 *}$, KEMING ZHANG $^{3 *}$, WEI LI $^{1}$, CHANGZHEN LIU $^{1}$, YU ZHANG ${ }^{1}$, \\ GANLIN ZHANG ${ }^{4}$, PAN MA ${ }^{1}$, LEI WANG ${ }^{1}$, GE ZHANG $^{1}$, HUA TAO $^{1}$ and BIN GAO ${ }^{1,5}$ \\ ${ }^{1}$ CAS Key Laboratory of Pathogenic Microbiology and Immunology (CASPMI), Centre for Molecular Immunology, \\ Institute of Microbiology, Chinese Academy of Sciences, Beijing; ${ }^{2}$ Key Laboratory of Infection and Immunity, \\ Institute of Biophysics, Chinese Academy of Sciences, Beijing; ${ }^{3}$ Department of Hepatobiliary Surgery, \\ 302 Military Hospital of China, Beijing; ${ }^{4}$ Department of Oncology, Beijing Hospital of Traditional Chinese Medicine \\ affiliated to Capital Medical University, Beijing; ${ }^{5}$ China-Japan Joint Laboratory of Molecular Immunology \\ and Microbiology, Institute of Microbiology, Chinese Academy of Sciences, Beijing, P.R. China
}

Received July 1, 2014; Accepted August 18, 2014

DOI: $10.3892 /$ ijo.2014.2663

\begin{abstract}
Targeting HER2 overexpressed breast cancer cells with anti-HER2 monoclonal antibodies inhibits tumor growth. Here we investigated whether HER2 can serve as a target for $\mathrm{T}$ cell-mediated immunotherapy of human colorectal carcinoma. Specific cytolytic activity of activated T cells (ATCs) armed with anti-CD3 $\mathrm{x}$ anti-HER2 bispecific antibody (HER2Bi-Ab) against HER2 ${ }^{+}$tumor cells was evaluated by bioluminescent signal generated by luciferase reporter on tumor cells in vitro and in vivo. In contrast to unarmed ATCs, increased cytotoxic activity of HER2Bi-armed ATCs against HER2+ tumor cells was observed. Moreover, HER2Bi-armed ATCs expressed higher level of activation marker CD69 and secreted significantly higher levels of IFN- $\gamma$ than the unarmed ATC counterpart.
\end{abstract}

Correspondence to: Dr Juan Ma or Professor Bin Gao, CAS Key Laboratory of Pathogenic Microbiology and Immunology (CASPMI), Centre for Molecular Immunology, Institute of Microbiology, Chinese Academy of Sciences, 1 Beichen West Road, Beijing 100101, P.R. China

E-mail: majuan96@sina.com

E-mail: bgao2004@gmail.com

${ }^{*}$ Contributed equally

Abbreviations: CRC, colorectal cancer; ATCs, activated T cells; VEGF, vascular endothelial growth factor; EGFR, epidermal growth factor receptor; HER2Bi-Ab, anti-CD3 x anti-HER2 bispecific antibody; PBMCs, peripheral mononuclear blood cells; ANOVA, one-way analysis of variance; TAA, tumor-associated antigen

Key words: HER2, colorectal carcinoma, bispecific antibody, immunotherapy
In addition, compared with anti-HER2 mAb (Herceptin ${ }^{\circledR}$ ) or unarmed ATC, HER2Bi-armed ATCs showed significant suppression against colorectal carcinoma cells. In colorectal tumor cell xenograft mice, infusion of HER2Bi-armed ATCs successfully inhibited the growth of Colo205-luc cells. The HER2Bi-armed ATCs with anti-tumor effects may provide a promising immunotherapy for colorectal carcinoma in the future.

\section{Introduction}

Colorectal cancer (CRC) is the third most frequent cancer worldwide and a common cause of cancer death primarily due to metastases that are resistant to conventional therapy (1). Over the last couple of years, a series of monoclonal antibodies targeting different tumor cell surface antigens have been tested in both experimental and clinical studies (2-5). Therapeutical antibodies have been successfully used for the treatment of metastatic CRC or after the failure of conventional chemotherapy, including cetuximab and panitumumab, antibodies to endothelial cell growth factor receptor (anti-EGFR), and bevacizumab, an antibody to vascular endothelial growth factor (anti-VEGF). Several drugs are under development with proven efficacy in phase II and III trials. However, the benefit of anti-EGFR therapy was limited to patients who had KRAS wild-type tumors with normal PTEN expression (6-8).

HER2 is a $185-\mathrm{kDa}$ transmembrane tyrosine kinase receptor, and belongs to the family of epidermal growth factor receptors (EGFRs), playing critical roles in epithelial cell growth and differentiation. HER2 overexpression has been found to be involved in pathogenesis of several human cancers (9-15). Although a dozen different ligands are known to bind to EGFRs, there is no ligand to be shown to bind directly to HER2. Instead, HER2 is thought to function as a co-receptor for other members of the ErbB/HER family, and frequently activated upon ligand binding to any of the other 
ErbB/HER receptors. Therefore, it is not surprising that HER2 has also been implicated in the development of carcinomas. In CRC the prevalence of HER-2/neu overexpression ranges from 0 to $83 \%$ (16-18), a big variation largely due to the lack of standardization of the detection methods. The success of HER-2/neu directed therapy in breast cancer suggests its potential role in the treatment of other tumors expressing HER2, including CRC. Herceptin ${ }^{\circledR}$ has been shown to inhibit colony formation of the HCA-7 colon cancer cell line and HCA-7 tumor xenografts (19).

In addition to the development of therapeutics for new targets, another approach to improve current antibody therapy was the development of bispecific antibodies. Cancer immunotherapies involving bispecific antibodies mediated-killing have been widely explored. Bispecific antibodies were first developed two decades ago and several molecules targeting different tumor cell surface antigens have been tested in clinical studies $(20,21)$. In this study, clinically approved anti-CD3 antibody was chemically conjugated with Herceptin antibody. The anti-CD3 $\mathrm{x}$ anti-HER2 bispecific antibody (HER2Bi-Ab) was then used to direct the activated T cells (ATCs) to kill colorectal carcinoma targets. Armed with HER2Bi-Ab, ATCs exhibited high levels of specific cytotoxicity and proliferation ability. The efficacy of HER2Bi-Ab for the inhibition of HER2-positive CRCs in SCID-Beige mouse model was also demonstrated.

\section{Materials and methods}

Cell lines and mice. Colo205-luc, HT-29-luc, BXPC-3-luc, PC-3M-luc, Hela-luc, and K562-luc cell lines were all from Caliper Life Sciences. The primary culture and the metastatic culture were derived from freshly isolated colon carcinoma cells from patients of primary colon carcinoma or hepatic metastasis of colonic carcinoma, respectively, in 302 Military Hospital of China. The consent was obtained from the patients before sample collection. The study complied with the Declaration of Helsinki and was approved by the Biomedical Research Ethics Committee of CAS Key Laboratory of Pathogenic Microbiology and Immunology. The agents for cell culture were all from Gibco Co. Beige-SCID mice (8-10 weeks) were purchased from Vital River Laboratories.

Isolation of peripheral blood lymphocytes and preparation and cryopreservation of activated T lymphocytes. Peripheral mononuclear blood cells (PBMCs) were isolated using Ficoll density gradient centrifugation from healthy donors supplied by the Beijing Blood Bank. PBMCs were cultured at $1 \times 10^{6} / \mathrm{ml}$ in RPMI-1640 medium supplemented with $10 \%$ FBS and $5 \mu \mathrm{g} / \mathrm{ml}$ anti-CD3 $\mathrm{mAb}$ and $100 \mathrm{IU} / \mathrm{ml}$ recombinant human IL-2 at $1 \times 10^{6} / \mathrm{ml}$. Half-volume medium exchange was performed every 3 days with medium containing fresh $100 \mathrm{IU} / \mathrm{ml}$ recombinant human IL-2 as the method previously described (22). On day 14, ATC expansion products of donors were on average $98.85 \pm 1.06 \% \mathrm{CD}^{+}$cells $(38.4 \pm 18.10 \%$ $\mathrm{CD}^{+}{ }^{+} \mathrm{CD} 4{ }^{+}$cells, and $66.35 \pm 9.83 \% \mathrm{CD}^{+}{ }^{+} \mathrm{CD} 8^{+} \mathrm{T}$ cells), the cells were used immediately or cryopreserved for further use. Based on an informed consent, this project was approved by the Biomedical Research Ethics Committee of CAS Key Laboratory of Pathogenic Microbiology and Immunology.
Synthesis of HER2Bi-Ab and arming of ATCs. Anti-HER2 (Herceptin ${ }^{\circledR}$; Roche) was reacted with sulfo-SMCC and anti-CD3 (OKT3; eBioscience; 85-16-0037-85) was reacted with Traut's reagents as previously described (23). Cryopreserved ATCs were thawed, and armed with HER2Bi at a concentration of $50 \mathrm{ng} / 10^{6}$ cells at room temperature for $30 \mathrm{~min}$ followed by washing the cells to eliminate unbound antibodies. The combination of OKT3 (50 ng/10 6 cells) and Herceptin $^{\circledR}\left(50 \mathrm{ng} / 10^{6}\right.$ cells $)$ pre-incubated ATCs were used as control unarmed ATCs.

Cytotoxicity assay. Cytotoxicity was measured with a luciferase quantitative assay (23-25). Target cells were seeded in duplicates in 96-well U-bottom microplates at $1 \times 10^{4} /$ well before the addition of HER2Bi-armed, or unarmed ATCs at various effector-to-target $(\mathrm{E} / \mathrm{T})$ ratios. Effector cells and tumor cells were allowed to interact at $37^{\circ} \mathrm{C}$ for $18 \mathrm{~h}$. A final concentration of $0.15 \mathrm{mg} / \mathrm{ml}$ D-luciferin (Synchem Chemie; Bc219-05) was added to each well.

IFN- $\gamma$ ELISA. Target cells were plated in 96-well U-bottom microplates at a concentration of $1 \times 10^{4} /$ well at $37^{\circ} \mathrm{C}$ overnight. HER2Bi-armed, or unarmed ATCs were then added at an $\mathrm{E} / \mathrm{T}$ ratio of 5:1 to target cells and incubated for $18 \mathrm{~h}$. The cell free supernatants were collected, and the IFN- $\gamma$ production was measured by using a human IFN- $\gamma$ ELISA kit (Thermo Scientific) according to the manufacturer's instructions.

Flow cytometric analysis. The anti-CD69-PE, anti-CD3-FITC, anti-mouse IgG-FITC secondary antibodies were from eBioscience, and anti-human IgG-FITC secondary antibody was from Beijing Zhongshan Golden Bridge Biotechnology, Co., Ltd. The cells were assayed with a Guava EasyCyte flow cytometer (Guava Technologies, Inc.) and the data analysis was carried out with FlowJo software version 7.6.1 (Tree Star, Inc.).

In vitro cell proliferation assay. For evaluating Herceptin ${ }^{\circledR}$ on colorectal tumor cell proliferation in vitro, colon carcinoma cells were seeded into 96-well plates in triplicates and incubated with the fresh medium or Herceptin ${ }^{\circledR}$ at the indicated concentration for $72 \mathrm{~h}$. For evaluating HER2Bi-armed ATCs on colorectal tumor cell proliferation in vitro, HT29-luc were seeded $\left(2 \times 10^{4} /\right.$ well) into 96-well plates in triplicates and allowed to adhere overnight. The following day, the medium was removed, and fresh medium alone or containing the unconjugated mAbs (50 ng/ml), ATCs $\left(2 \times 10^{5} /\right.$ well $)$, HER2Bi-armed ATCs $\left(2 \times 10^{5} /\right.$ well, armed with $\left.50 \mathrm{ng} / \mathrm{HER} 2 \mathrm{Bi} / 10^{6} \mathrm{ATCs}\right)$ or unarmed ATCs was added to wells. Cultures were incubated for $18 \mathrm{~h}$, then medium was removed and $100 \mu \mathrm{l}$ of fresh serum-free medium containing 1/10 (v/v) Cell Counting kit-8 (CCK8; Dojindo Laboratories) reagent was added to each well and incubated for an additional $3 \mathrm{~h}$. After incubation, the absorbancy of colorectal tumor cells was measured using a 96 -well plate reader at $450 \mathrm{~nm}$. Cell proliferation was assessed by the absorbance values according to the manufacturer's protocol.

In vivo tumor inhibition studies. In tumor prevention studies, Colo205-luc cells $\left(1 \times 10^{6} /\right.$ mouse) were mixed with 
Colo205-luc

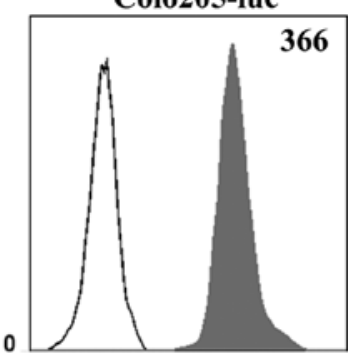

PC-3M-luc

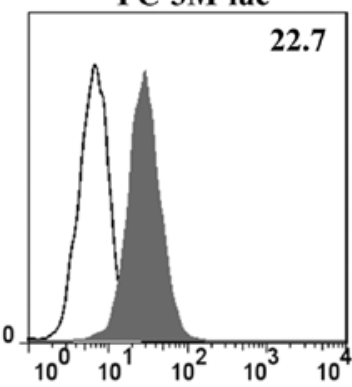

HT29-luc

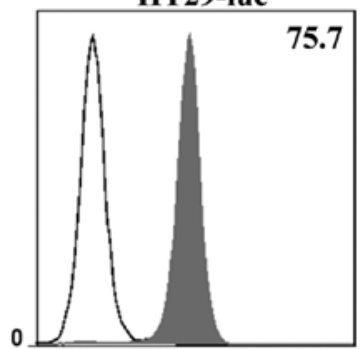

BXPC3-luc

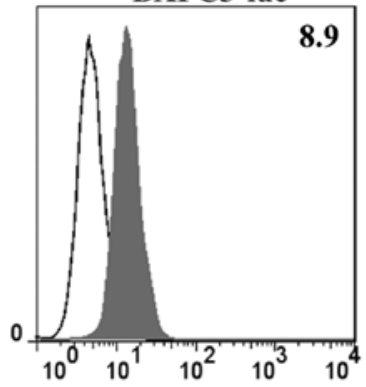

Hela-luc

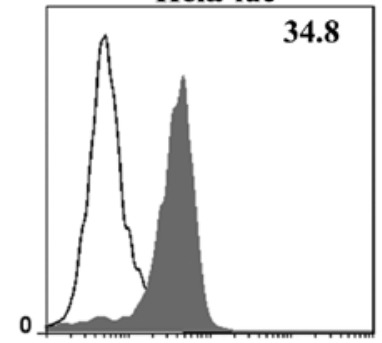

K562-luc

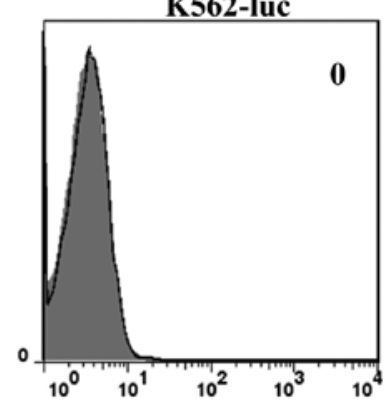

Figure 1. Expression of HER2 on different human tumor cells. HER2/neu-expressing profiles of tumor cell lines by flow cytometry. Shaded histogram represents cells stained with Herceptin ${ }^{\circledR}$ and un-shaded histogram represents cells stained with control human IgG. Mean fluorescent intensity (MFI) values obtained with Herceptin ${ }^{\circledast}$ staining subtract the control staining are indicated in the upper right of the histogram.

\section{A a}

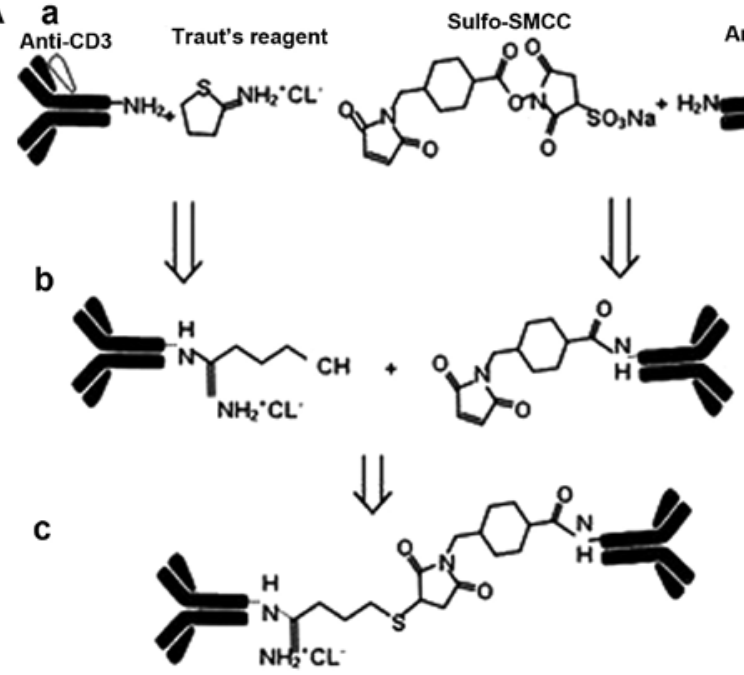

C

HER2 $^{+}$cell

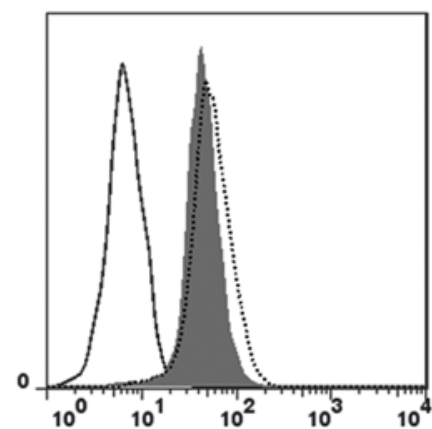

D

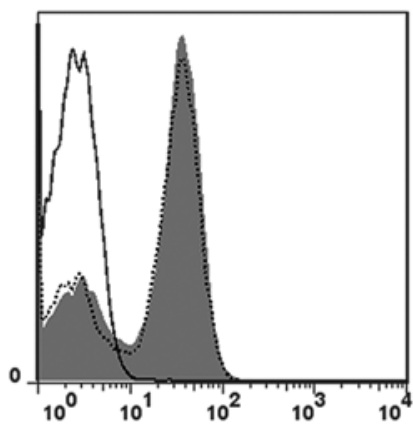

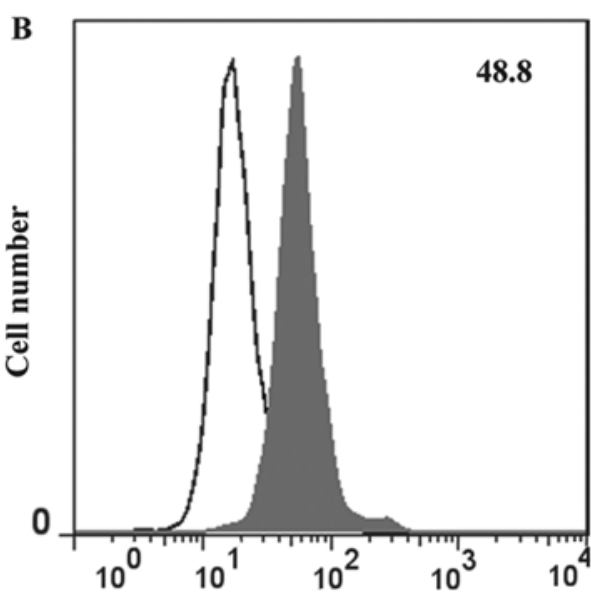

anti-HER2 $x$ anti-CD3

E HER2-CD3- cell

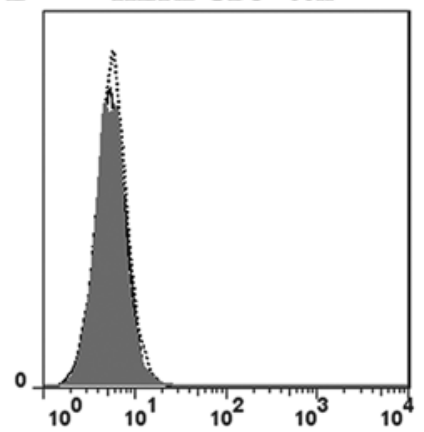

Figure 2. General scheme for the generation of anti-CD3 x anti-HER2 bispecific antibody (HER2Bi-Ab). (A) General scheme for the generation of HER2Bi-Ab. (B) Flow cytometry based binding assay for HER2Bi-Ab. Colo205-luc cells were incubated with HER2Bi-Ab (shaded histogram) or combination of OKT3 and Herceptin $^{\circledR}$ (the black line), HER2Bi-Ab binding was evaluated by FITC goat anti-mouse IgG to detect the anti-CD3 moiety of the HER2Bi-Ab. (C) HT29-luc cells were incubated with HER2Bi-Ab (shaded histogram), Herceptin ${ }^{\circledR}$ (dot histogram), or control IgG (open histogram), HER2Bi-Ab binding was analyzed by FITC goat anti-human IgG to detect the anti-HER2 moiety of the HER2Bi-Ab. (D) Peripheral mononuclear blood cells (PBMCs) were incubated with HER2Bi-Ab (shaded histogram), OKT3 (dot histogram), or control IgG (open histogram), the HER2Bi-Ab binding was analyzed by FITC goat anti-mouse IgG to detect the anti-CD3 moiety of the HER2Bi-Ab. (E) K562cells were incubated with HER2Bi-Ab (shaded histogram), OKT3 (dot histogram), or control IgG (open histogram), the HER2Bi-Ab binding was analyzed by FITC-goat-anti-mouse IgG to detect the anti-CD3 moiety of the HER2Bi-Ab. 

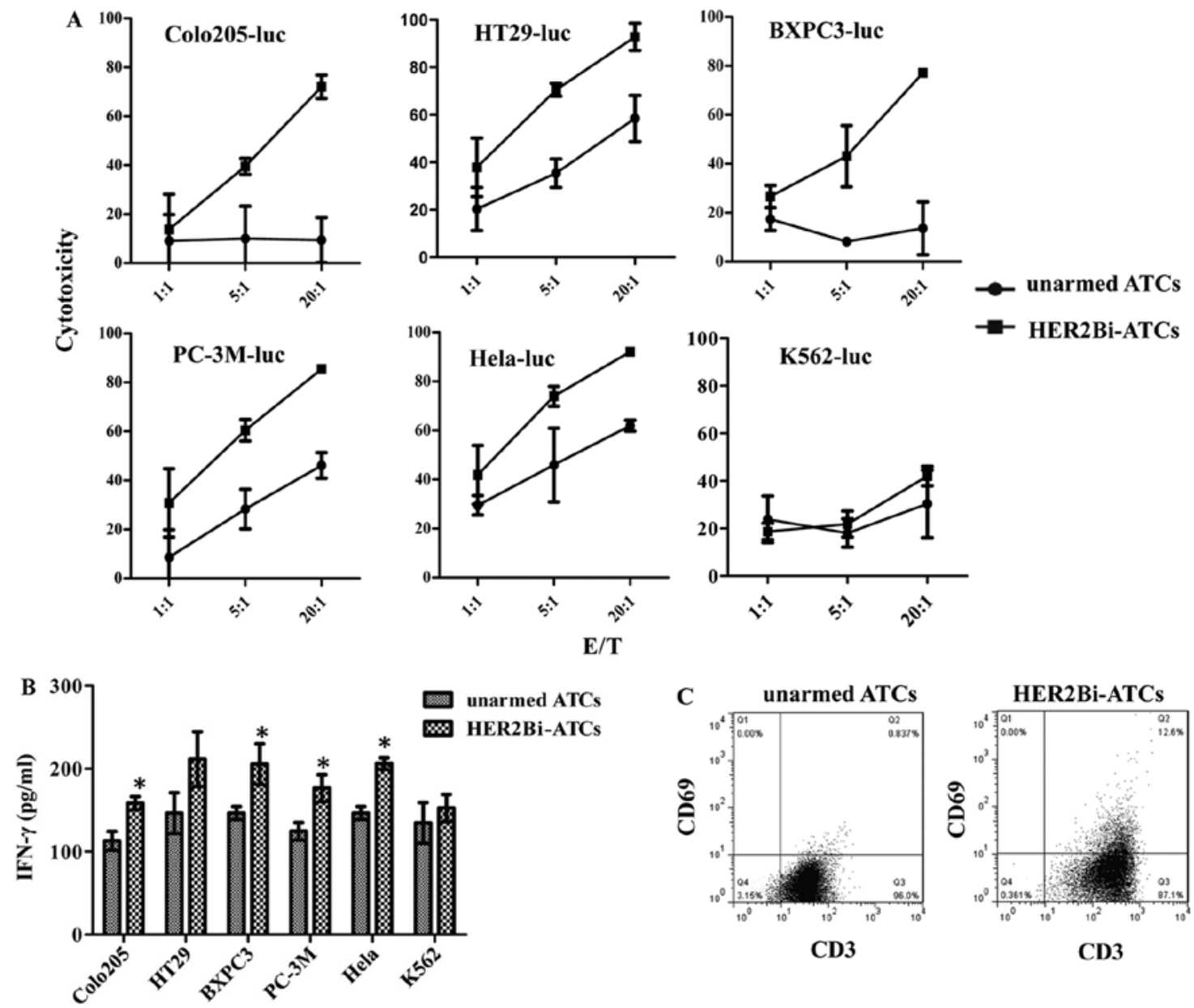

Figure 3. The antitumor effect of HER2Bi-armed activated T cells (ATCs) against different tumor cell lines. (A) Target cells were incubated either with HER2Bi-armed ATCs or a combination of OKT3 and Herceptin with ATCs (unarmed ATCs) for $18 \mathrm{~h}$, luciferase quantitative assay was performed to determine cytotoxicity against different target cells at different effector-to-target (E/T) ratios. (B) IFN- $\gamma$ secretion by HER2Bi-armed ATCs against different target tumor cells compared with unarmed ATCs. Supernatants of co-cultures at E/T ratio of 5:1 were harvested at $18 \mathrm{~h}$ and analyzed for IFN- $\gamma$ level. The data are mean \pm SD of triplicate determination. Shown is a representative experiment of three. (C) Expression of CD69 on HER2Bi-armed ATCs or unarmed ATCs was detected by flow cytometry after $18 \mathrm{~h}$ co-culture with HT-29-luc cell at E/T ratio of 5:1. $\mathrm{P}<0.05$, HER2Bi-armed ATCs compared with unarmed ATCs under similar conditions.

HER2Bi-armed ATCs (1x10 $/$ mouse) or unarmed-ATCs. The cell mixtures were immediately inoculated subcutaneously on the rear flank of five SCID-Beige mice per group. In tumor growth delay studies, SCID-Beige mice ( $\mathrm{n}=5$ mice per group) were injected i.p. with $3 \times 10^{6}$ Colo205-luc cells. Subsequently, HER2Bi-armed ATCs $\left(3 \times 10^{7} /\right.$ mouse) or control ATCs were administered i.p. on day 3, 10, and 17. In order to follow up the tumor growth, in vivo bioluminescence imaging was operated on the indicated days for 4 weeks. Bioluminescent imaging was taken using Xenogen IVIS-100 imaging system with Living Image software (Caliper Life Sciences). The signal intensity of tumor burdens was expressed as total photons $/ \mathrm{sec} / \mathrm{cm}^{2}\left(\mathrm{p} / \mathrm{sec} / \mathrm{cm}^{2} / \mathrm{sr}\right)$.

Statistical analysis. All experiments were repeated at least twice and mostly three times. Data were analyzed using Graphpad Prism 5 software, the data are presented as the means \pm SD. Unpaired Student's t-test (two-tailed) or the Mann-Whitney test was used for comparison of two groups where appropriate. One-way analysis of variance (ANOVA) followed by Dunnett's post hoc for multiple comparison.
$\mathrm{P}<0.05$ was considered as statistically significant. The number with a significant difference from a control is denoted by an asterisk in the figures.

\section{Results}

HER2 overexpression in human CRC cells. The surface expression of HER2 on human tumors from different tissue origins was assessed by FACS analysis including CRC (Colo205-luc and HT-29-luc), pancreatic cancer (BXPC3-luc), prostate cancer (PC-3M-luc), cervix cancer (Hela-luc), and leukemia (K652-luc). As shown in Fig. 1, HER2 expression measured as mean fluorescent intensity (MFI) in CRC cells (Colo205-luc: 366; HT29-luc: 75.7) was much higher than that in other tumor cells (BXPC3-luc: 8.9; PC-3M-luc: 22.7; Hela-luc: 34.8). HER2 was not detected on K562 cells used as a negative control.

Preparation and characterization of HER2Bi and ATCs. Herceptin ${ }^{\circledR}$ antibody was hetero-conjugated with OKT3 chemically and named as HER2Bi (Fig. 2A). The binding 
A

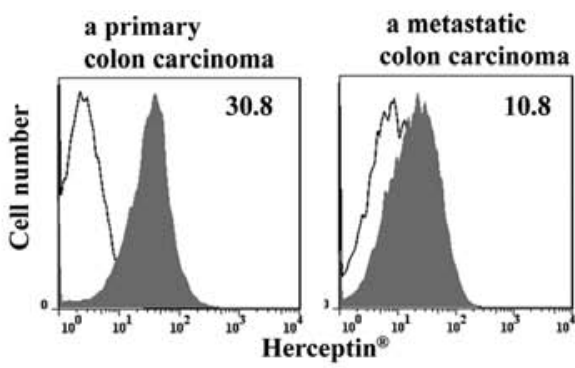

unarmed ATCs
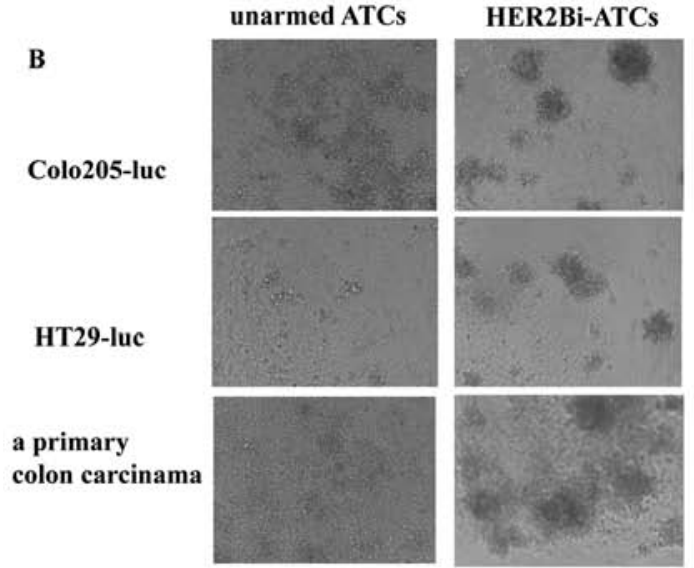

a metastatic colon carcinama
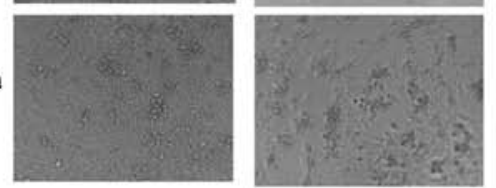

C
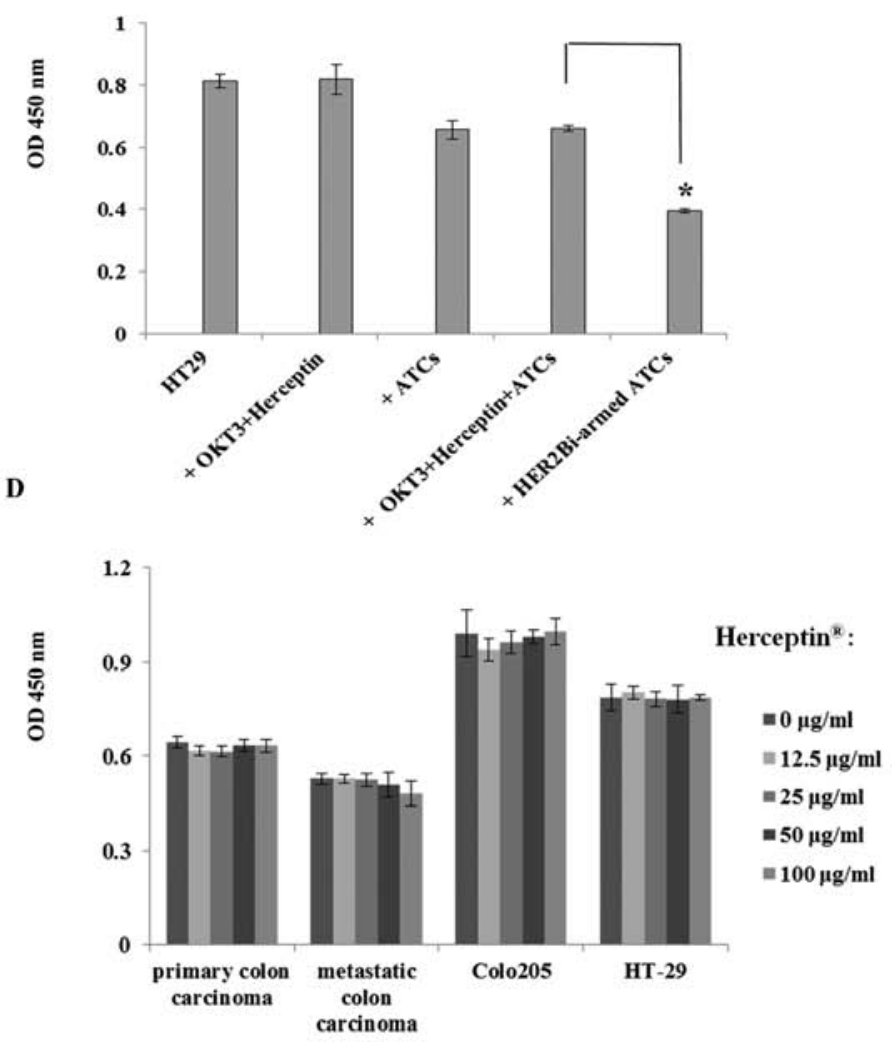

Figure 4. Anti-proliferative effects of Herceptin ${ }^{\circledast}$ or HER2Bi-armed activated T cells (ATCs) against colorectal carcinoma cells. (A) Expression of HER2 on a human primary (left) and a metastatic (right) colon carcinoma cell culture. Shaded histogram represents cells stained with Herceptin ${ }^{\circledR}$ and un-shaded

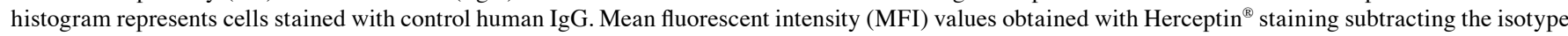
control staining are indicated in the upper right of the histogram. (B) Target colorectal carcinoma cells were incubated either with HER2Bi-armed ATCs or unarmed ATCs for $18 \mathrm{~h}$ at effector-to-target (E/T) ratio of 10:1, and real-time photographs were taken at x200 magnification. (C) Growth inhibition of colorectal carcinoma cells by HER2Bi-armed ATCs. Adherent HT29-luc cells were incubated either with unconjugated OKT3 and Herceptin, or ATCs, or HER2Bi-armed ATCs, or unarmed ATCs for $18 \mathrm{~h}$. The proliferation of HT29-luc cells was assessed by Cell Counting kit-8 (CCK8) assay with the OD value measured at $450 \mathrm{~nm}$. (D) Effects of Herceptin ${ }^{\circledast}$ on the proliferation of colorectal carcinoma cells. Colorectal carcinoma cells were treated with or without Herceptin ${ }^{\circledast}$ for $72 \mathrm{~h}$ and the proliferation of cells was assessed by CCK8 assay with the OD value was measured at $450 \mathrm{~nm}$. The data are mean \pm SD of triplicate experiments, and a representative experiment of three is shown. "P $<0.05$, HER2Bi-armed ATCs compared with unarmed ATCs under similar conditions.

specificity of HER2Bi against HER2 was tested. Colo205-luc cells were first stained by HER2Bi, then an anti-mouse IgG-FITC was added to detect the CD3 moiety of HER2Bi-Ab. Only functionally bispecific HER2Bi antibody was able to bind to Colo205-luc cells by HER2 recognized Herceptin ${ }^{\circledR}$ and be detected through mouse origin OKT3 by anti-mouse secondary antibody. As shown in Fig. 2B, positively stained cells were detected in $91.3 \%$ of the Colo205-luc population with an MFI of 48.8. Moreover, binding of HER2Bi-Ab on HER $2^{+}$cells was confirmed by FITC goat anti-human IgG to detect the anti-HER2 moiety of the HER2Bi-Ab (Fig. 2C). To evaluate the binding of HER $2 \mathrm{Bi}-\mathrm{Ab}$ to $\mathrm{CD}^{+}$cells, $\mathrm{PBMC}$ were incubated with HER2Bi-Ab, and the binding of HER2Bi-Ab to $\mathrm{CD}^{+}$cells was evaluated by FITC goat anti-mouse $\operatorname{IgG}$ to detect the anti-CD3 moiety of the HER2Bi-Ab (Fig. 2D). In contrast, HER2Bi-Ab did not bind to CD3-HER2-K652 cells (Fig. 2E).

Cytotoxicity of HER2Bi-armed ATC with IFN- $\gamma$ production on different tumor cell lines. The amount of HER2Bi required to arm ATCs ranged from 5 to $500 \mathrm{ng} / 10^{6}$ cells.
Since 50 and $500 \mathrm{ng} / 10^{6}$ cells showed similar cytotoxicity, we chose $50 \mathrm{ng} / 10^{6}$ ATCs as the concentration of HER2Bi for all subsequent experiments, and ATCs mixed with both individual OKT3 and Herceptin ${ }^{\circledR}$ were used as unarmed ATC control. Cytotoxic effects of HER2Bi-armed ATCs on different HER $2^{+}$tumor cells were tested in vitro. The assays were performed at $\mathrm{E} / \mathrm{T}$ ratios of 1:1, 5:1 and 20:1. After $18 \mathrm{~h}$ incubation with HER2Bi-armed ATCs or unarmed ATCs, bioluminescence imaging signal in tumor cells expressed in photons per second was converted into living cell number and the cytotoxicity assays was calculated at the indicated E/T ratios. As shown in Fig. 3A, the percentage of cytotoxicity with armed ATCs was significantly greater than that with unarmed effectors at E/T ratio of 5:1 and 20:1 in Colo205-luc, HT29-luc, BXPC-3, PC-3M-luc, and Hela-luc cells.

To analyze the cytokines along with the cytotoxicity, supernatants of cell cultures were analyzed for IFN- $\gamma$ production at $\mathrm{E} / \mathrm{T}$ ratio of 5:1. As shown in Fig. 3B, significant increase was observed for IFN- $\gamma$ secretion by HER2Bi-armed ATCs over their unarmed ATC counterparts when ATCs were co-cultured with Colo205-luc, BXPC3-luc, PC-3M-luc 
A
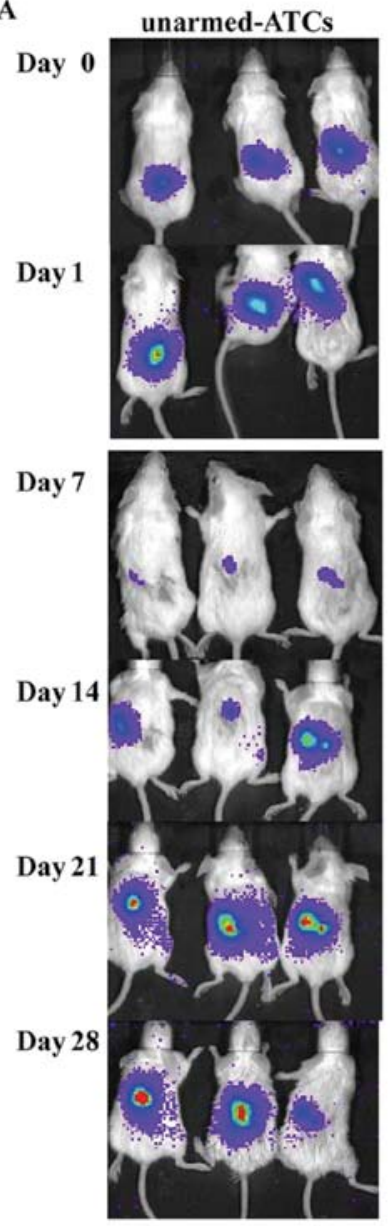

HER2Bi-ATCs
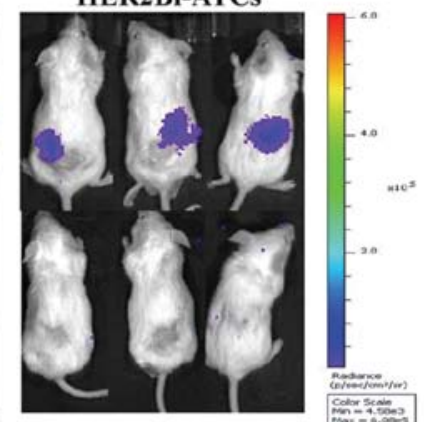

B

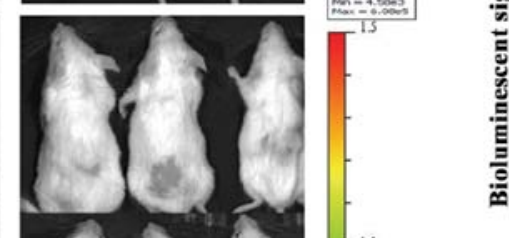

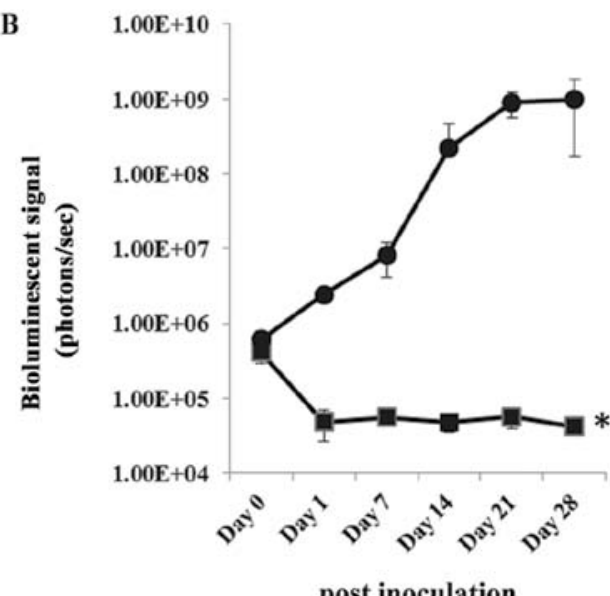

post inoculation

$\rightarrow$ unarmed ATCs

HER2Bi-ATCs

Figure 5. Prevention effects of HER2Bi-armed activated T cells (ATCs) to colorectal tumor cells in vivo. SCID-Beige mice were inoculated with Colo205-luc cells mixed with HER2Bi-ATCs or unarmed-ATCs, respectively, on day 0 , each group contains $n=5$ mice. (A) Bioluminescence images of three representative mice of each group are shown on the indicated day. (B) Images were analyzed using Living Image software and the tumor values represented as total flux measurements in photons $/ \mathrm{sec}$, mean values of tumor growth curves are shown. ${ }^{*} \mathrm{P}<0.05$.

or Hela-luc cells, respectively $(\mathrm{P}<0.05)$. Moreover, FACS analysis of HER2Bi-armed ATCs showed an increased CD69 expression over their unarmed ATCs counterparts (Fig. 3C).

Cells derived from both primary and metastatic human colorectal carcinoma were tested to evaluate whether they also expressed high levels of HER2 proteins. As shown in Fig. 4A, HER2-positive stained cells were detected by FACS analysis in $90 \%$ of the primary colorectal carcinoma cell population with an MFI of 30.8 and in $50 \%$ of the metastatic colorectal carcinoma cell population with an MFI of 10.8. Then, HER2Bi-armed ATCs were tested for cytotoxicity on HER2 positive primary cells derived from colorectal carcinoma. The assays were performed at E/T ratio of 10:1. After $18 \mathrm{~h}$ incubation with HER2Bi-armed ATCs or unarmed ATCs, as shown in Fig. 4B, real-time photographs of each colorectal carcinoma group were taken at $\mathrm{x} 200$ magnification. It was demonstrated that HER2Bi-ATCs, but not equivalent number of unarmed-ATCs, aggregated with all the four colorectal carcinoma cell types, clustering around the edge of targeting cell bulk, which showed the specific lysis of HER2Bi-ATCs.

The growth inhibition of colorectal carcinoma cells by HER2Bi-armed ATCs. Furthermore, HER2Bi-armed ATCs were tested for the inhibitory efficacy on HER 2 positive colorectal carcinoma. In cell proliferation assay, unconjugated mAbs (OKT3 and Herceptin ${ }^{\circledR}$ ), ATC alone, a combination of OKT3 and Herceptin ${ }^{\circledR}$ with ATC (unarmed ATC), or HER2Bi-armed ATC (E/T ratio of 10:1) were co-cultured with HT29-luc cells for $18 \mathrm{~h}$, respectively. As expected, HER2Bi-armed ATCs showed a superior growth inhibition on colorectal carcinoma cells, compared to the other groups (Fig. 4C). Unexpectedly, even at the concentration of $100 \mu \mathrm{g} / \mathrm{ml}$, Herceptin ${ }^{\circledast}$ did not inhibit the proliferation of colorectal tumor cells after $72 \mathrm{~h}$ incubation in vitro (Fig. 4D).

HER2Bi-armed ATCs inhibited Colo205 tumor growth in SCID-Beige mice. To determine whether HER2Bi-armed ATCs could prevent tumor growth in vivo, SCID-Beige mice were engrafted subcutaneously with Colo205-luc cells. The growth of tumor was monitored with bioluminescent imaging. In Fig. 5A, results of three representative mice of each group are shown. When mice were co-injected with unarmed-ATCs, the light signal increased over time from day 1 to 28 . In contrast, the signal disappeared on day 1 and vanished completely from day 7 to 28 when mice were co-injected with HER2Bi-armed ATCs. Once injected, mice were given no further treatment but were monitored weekly for tumor development up through day 28 following initial injection. The mean bioluminescence 
Colo205-luc only

Control-ATCs

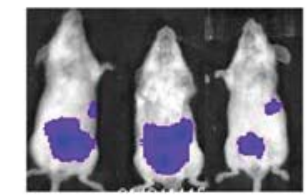

HER2Bi-ATCs

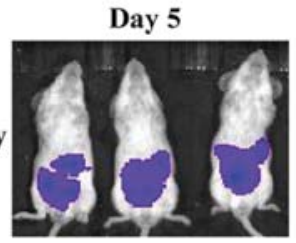

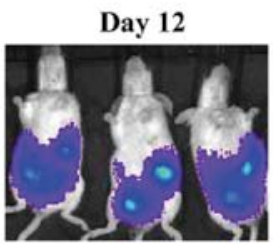
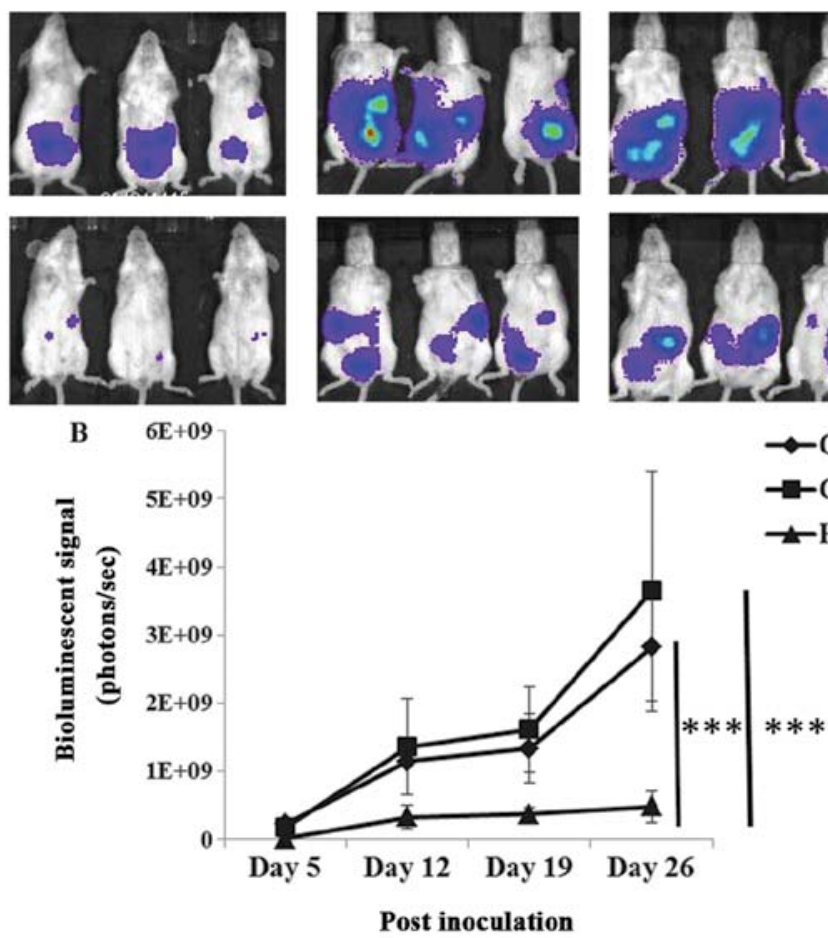
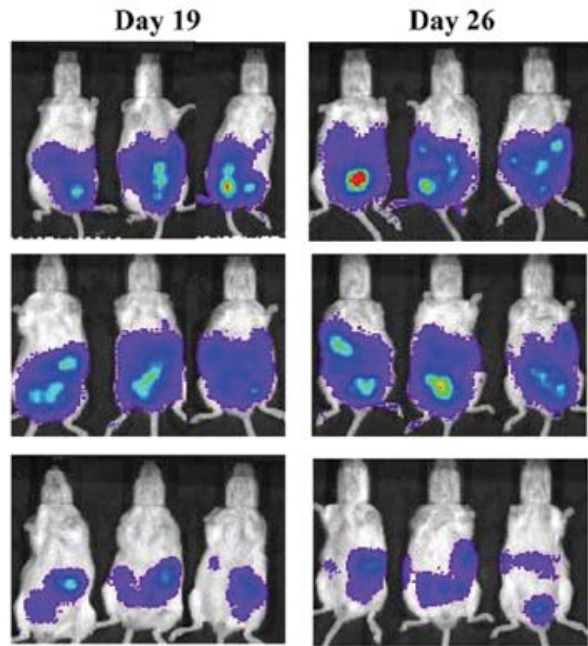

$\rightarrow$ Colo205 only

- -Control-ATCs

-Her2Bi-ATCs

-

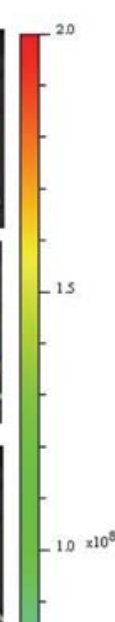

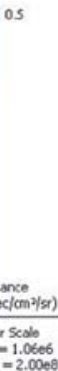

Figure 6. Suppression effects of HER2Bi-armed activated T cells (ATCs) to colorectal tumor cells in vivo. SCID-Beige mice were inoculated with Colo205-luc cells i.p. on day 0, and 3 days later HER2Bi-ATCs or control ATCs were administered i.p. respectively, each group contains n=5 mice. (A) Bioluminescence images of three representative mice of each group are shown on the indicated day. (B) Images were analyzed using Living Image software and tumor values represent as total flux measurement in photons/sec, mean values of tumor growth curves are shown. ${ }^{* * *} \mathrm{P}<0.0001$.

signal of each test group correlated with the number of living Colo205-luc cells as shown in Fig. 5B.

To further determine whether HER2Bi-armed ATCs could suppress tumor growth in vivo, SCID-Beige mice were engrafted intraperitoneally with Colo205-luc cells. Three days later, mice were treated with HER2Bi-armed ATCs or control ATCs weekly three times. The growth of tumor was monitored with bioluminescent imaging. In Fig. 6A, three representative mice of each group are shown. When Colo205-luc cells were inoculated alone, light signal increased over time. A similar kinetics of tumor growth was shown in mice that were injected with control ATCs. As for mice treated with HER2Bi-armed ATCs, the signal diminished from day 5 to 26 compared with other two groups. The mean bioluminescence signal of each test group correlated with the number of living Colo205-luc cells was shown in Fig. 6B. HER2Bi-armed ATCs inhibited the tumor growth significantly, whereas control ATCs did not, at every check point.

\section{Discussion}

Therapeutic antibodies such as bevacizumab, cetuximab, and panitumumab significantly improve survival rates of patients with advanced CRC. However, due to the limitations of conventional antibodies, including the redundancy in antibody targeted molecular pathways, inadequate exposure of the tumor due to the antibody limited tissue distribution, and immunogenicity caused by repeatable treatment, alternative approaches to improve current antibody strategy are urgently needed. One improvement is the use of $\mathrm{T}$ cell directed bispecific antibodies, comprising of an anti-CD3 $\mathrm{mAb}$ hetero-conjugated to a different $\mathrm{mAb}$ specific to a selected tumor-associated antigen (TAA). Such a bispecific antibody will make every $\mathrm{T}$ cell TAA-specific to redirect $\mathrm{T}$ cells to target tumor cells. HER2 is an ideal candidate used as a target by various tumor imaging and antibody-based therapeutic approaches. Phase I/II immunotherapy with HER2Bi-Ab and/or armed ATCs are currently ongoing in women with metastatic breast cancer $(26,27)$.

In this study, we tested whether HER 2 is a useful target for the development of novel bispecific $\mathrm{Ab}$ therapeutics in CRC, and examined in vitro and in vivo antitumor effects of HER2Bi-armed ATCs. Our study findings are relevant to the therapeutic application of target HER2 against CRC. The high expression of HER2 in colorectal carcinoma was confirmed by FACS analysis. In addition, primary or metastatic colon carcinoma cell cultures were also shown to express high level of HER2 antigen. In our present study HER2Bi-armed ATCs provided significant anti-proliferative and cytotoxic activity against HER2-positive colorectal carcinoma cells although 
anti-HER2 antibody alone had no inhibitory effect to colorectal carcinoma cells tested in vitro. Additionally, HER2Bi-armed ATCs expressed higher level of activation marker CD69 and secreted a higher level of IFN- $\gamma$ than unarmed ATC counterpart against colorectal carcinoma target cells. Furthermore, infusion of HER2Bi-armed ATCs remarkably inhibited the growth of colorectal carcinoma cells in the xenograft mouse tumor model.

Our results have shown that ATCs armed with HER2Bi released cytokine IFN- $\gamma$ upon incubation with the tumor cells. The increased secretion of cytokine demonstrated that ATCs were being reactivated upon binding to tumor cells. IFN- $\gamma$ secreted by HER2Bi-armed ATCs in the presence of tumor may not only cause direct tumor killing but also serve to modulate immune networks to induce local and/or systemic immune responses to tumors, capable of counteracting tumor-induced suppression by TGF- $\beta$, IL- 4 , and IL-10 $(28,29)$. Flow cytometry results provided evidence that HER2Bi-armed ATCs expressed higher level of CD69 than the unarmed-ATC counterparts. CD69 represents a marker of early T-cell activation and acts as a costimulatory molecule that increased T-cell responses following TCR-ligand interaction (30). Therefore, IFN- $\gamma$ produced by HER2Bi-armed ATC upon its binding to tumor antigen may be clinically beneficial. HER2Bi-Ab did not bind to CD3-HER2-K562 cells, confirming the specificity of the HER2Bi-Ab.

In tumor growth delay studies, HER2Bi-armed ATCs prevented and furthermore significantly inhibited tumor growth in mice bearing established HER2-positive Colo205 xenografts, whereas control unarmed ATCs did not, confirming the specificity of the targets. Similarly, in a pilot study, HER2Bi-armed ATCs injected intratumorally induced remission of human hormone-refractory prostate tumor in severe combined immunodeficient mice (31). Conceivably, the binding of the effector cells at the tumor site by armed ATCs may not only augment tumoricidal activity but also increase local cytokine secretion leading to the recruitment of other immune effectors $(32,33)$.

Although clinical studies have shown that Herceptin ${ }^{\circledR}$ significantly improves the overall survival of breast cancer patients, an unforeseen significant side-effect of cardiotoxicity manifested as left ventricular dysfunction and heart failure (34). In our study, HER2Bi-armed ATCs were highly effective in eliminating tumor cells both in vitro and in vivo at very low concentration of HER2-Bi-Ab. Also, our studies showed Herceptin ${ }^{\circledR}$ failed to inhibit proliferation of colon carcinoma cells in vitro, partly because of the HER2 expression on colon carcinoma cell lines were barely middle or low compared with the HER2 overexpressing breast cancer cell line SKBR3 (35). Therefore, more importantly, our study provided a new strategy for treatment of colon cancer in the event when the expression of the target tumor antigen e.g., HER2, is not high.

In conclusion, taken together with the in vitro cytotoxicity and cytokine secretion studies, the ability of HER2Bi-armed ATCs to prevent the development and suppress the growth of tumors in xenograft mice suggests that HER2Bi-armed ATCs could be used as a good strategy for the treatment of $\mathrm{HER}^{+} \mathrm{CRC}$ patient and produce clinically significant antitumor effects.

\section{Acknowledgements}

This study was funded by the grants from the Basic Research Program of China (973 Program, No. 2013CB531502), the Ministry of Science and Technology of China (S\&T major Program: No. 2012ZX1004701-001-002), and the National Nature Science Foundation of China (No. 31370889 and 31170829).

\section{References}

1. Jochems C and Schlom J: Tumor-infiltrating immune cells and prognosis: the potential link between conventional cancer therapy and immunity. Exp Biol Med (Maywood) 236: 567-579, 2011.

2. Xiang J, Pan Z, Attah-Poku S, Babiuk L, Zhang Y and Liu E: Production of hybrid bispecific antibody recognizing human colorectal carcinoma and CD3 antigen. Mol Biother 4: 15-23, 1992.

3. Gautherot E, Rouvier E, Daniel L, Loucif E, Bouhou J, Manetti C, Martin M, Le Doussal JM and Barbet J: Pretargeted radioimmunotherapy of human colorectal xenografts with bispecific antibody and 131I-labeled bivalent hapten. J Nucl Med 41: 480-487, 2000.

4. Herrmann I, Baeuerle PA, Friedrich M, Murr A, Filusch S, Rüttinger D, Majdoub MW, Sharma S, Kufer P, Raum T and Münz M: Highly efficient elimination of colorectal tumor-initiating cells by an EpCAM/CD3-bispecific antibody engaging human T cells. PLoS One 5: e13474, 2010.

5. Kim DD and Eng C: The promise of mTOR inhibitors in the treatment of colorectal cancer. Expert Opin Investig Drugs 21: 1775-1788, 2012.

6. Misale S, Yaeger R, Hobor S, Scala E, Janakiraman M, Liska D, ValtortaE, Schiavo R, Buscarino M, Siravegna G, et al: Emergence of KRAS mutations and acquired resistance to anti-EGFR therapy in colorectal cancer. Nature 486: 532-536, 2012.

7. Tol J and Punt CJ: Monoclonal antibodies in the treatment of metastatic colorectal cancer: a review. Clin Ther 32: 437-453, 2010.

8. Wang ZH, Gao QY and Fang JY: Loss of PTEN expression as a predictor of resistance to anti-EGFR monoclonal therapy in metastatic colorectal cancer: evidence from retrospective studies. Cancer Chemother Pharmacol 69: 1647-1655, 2012.

9. Ross JS, Slodkowska EA, Symmans WF, Pusztai L, Ravdin PM and Hortobagyi GN: The HER-2 receptor and breast cancer: ten years of targeted anti-HER-2 therapy and personalized medicine. Oncologist 14: 320-368, 2009.

10. Hillig T, Thode J, Breinholt MF, Franzmann MB, Pedersen C, Lund F, Mygind H, Sölétormos G and Rudnicki M: Assessing HER2 amplification by IHC, FISH, and real-time polymerase chain reaction analysis (real-time PCR) following LCM in formalin-fixed paraffin embedded tissue from 40 women with ovarian cancer. APMIS 120: 1000-1007, 2012.

11. Janjigian YY, Werner D, Pauligk C, Steinmetz K, Kelsen DP, Jäger E, Altmannsberger HM, Robinson E, Tafe LJ, Tang LH, Shah MA and Al-Batran SE: Prognosis of metastatic gastric and gastroesophageal junction cancer by HER2 status: a European and USA International collaborative analysis. Ann Oncol 23: 2656-2662, 2012.

12. Jørgensen JT and Hersom M: HER2 as a prognostic marker in gastric cancer-a systematic analysis of data from the literature. J Cancer 3: 137-144, 2012.

13. Takenaka M, Hanagiri T, Shinohara S, Kuwata T, Chikaishi Y, Oka S, Shigematsu Y, Nagata Y, Shimokawa H, Nakagawa M, Uramoto H, So T and Tanaka F: The prognostic significance of HER 2 overexpression in non-small cell lung cancer. Anticancer Res 31: 4631-4636, 2011.

14. Bergmann F, Moldenhauer G, Herpel E, Gaida MM, Strobel O, Werner J, Esposito I, Müierköster SS, Schirmacher P and Kern MA: Expression of L1CAM, COX-2, EGFR, c-KIT and Her2/neu in anaplastic pancreatic cancer: putative therapeutic targets? Histopathology 56: 440-448, 2010

15. Krähn G, Leiter U, Kaskel P, Udart M, Utikal J, Bezold G and Peter RU: Coexpression patterns of EGFR, HER2, HER3 and HER4 in non-melanoma skin cancer. Eur J Cancer 37: 251-259, 2001.

16. Schuell B, Gruenberger T, Scheithauer W, Zielinski $\mathrm{Ch}$ and Wrba F: HER 2/neu protein expression in colorectal cancer. BMC Cancer 6: 123, 2006. 
17. Kountourakis P, Pavlakis K, Psyrri A, Rontogianni D, Xiros N, Patsouris E, Pectasides D and Economopoulos T: Clinicopathologic significance of EGFR and Her-2/neu in colorectal adenocarcinomas. Cancer J 12: 229-236, 2006.

18. Kavanagh DO, Chambers G, O'Grady L, Barry KM, Waldron RP, Bennani F, Eustace PW and Tobbia I: Is overexpression of HER-2 a predictor of prognosis in colorectal cancer? BMC Cancer 9: $1,2009$.

19. Mann M, Sheng H, Shao J, Williams CS, Pisacane PI, Sliwkowski MX and DuBois RN: Targeting cyclooxygenase 2 and HER-2/neu pathways inhibits colorectal carcinoma growth. Gastroenterology 120: 1713-1719, 2001.

20. Fury MG, Lipton A, Smith KM, Winston CB and Pfister DG: A phase-I trial of the epidermal growth factor receptor directed bispecific antibody MDX-447 without and with recombinant human granulocyte-colony stimulating factor in patients with advanced solid tumors. Cancer Immunol Immunother 57: 155-163, 2008

21. Seimetz D, Lindhofer $H$ and Bokemeyer C: Development and approval of the trifunctional antibody catumaxomab (anti-EpCAM x anti-CD3) as a targeted cancer immunotherapy. Cancer Treat Rev 36: 458-467, 2010.

22. Clay TM, Custer MC, Sachs J, Hwu P, Rosenberg SA and Nishimura MI: Efficient transfer of a tumor antigen-reactive TCR to human peripheral blood lymphocytes confers anti-tumor reactivity. J Immunol 163: 507-513, 1999.

23. Ma J, Han H, Liu D, Li W, Feng H, Xue X, Wu X, Niu G, Zhang G, Zhao Y, Liu C, Tao $\mathrm{H}$ and Gao B: HER2 as a promising target for cytotoxicity $\mathrm{T}$ cells in human melanoma therapy. PLoS One 8: e73261, 2013.

24. Fu X, Tao L, Rivera A, Williamson S, Song XT, Ahmed N and Zhang X: Simple and sensitive method for measuring tumor-specific T cell cytotoxicity. PLoS One 5: e11867, 2010.

25. Brown CE, Wright CL, Naranjo A, Vishwanath RP, Chang WC, Olivares S, Wagner JR, Bruins L, Raubitschek A, Cooper LJ and Jensen MC: Biophotonic cytotoxicity assay for high-throughput screening of cytolytic killing. J Immunol Methods 297: 39-52, 2005.

26. Repp R, van Ojik HH, Valerius T, Groenewegen G, Wieland G, Oetzel C, Stockmeyer B, Becker W, Eisenhut M, Steininger $\mathrm{H}$, et al: Phase I clinical trial of the bispecific antibody MDX-H210 (anti-FcgammaRI x anti-HER-2/neu) in combination with Filgrastim (G-CSF) for treatment of advanced breast cancer. Br J Cancer 89: 2234-2243, 2003.
27. Lum LG, Rathore R, Cummings F, Colvin GA, Radie-Keane K, Maizel A, Quesenberry PJ and Elfenbein GJ: Phase I/II study of treatment of stage IV breast cancer with OKT3 $\mathrm{x}$ trastuzumab-armed activated $\mathrm{T}$ cells. Clin Breast Cancer 4: 212-217, 2003.

28. Sheu BC, Lin RH, Lien HC, Ho HN, Hsu SM and Huang SC: Predominant $\mathrm{Th} 2 / \mathrm{Tc} 2$ polarity of tumor-infiltrating lymphocytes in human cervical cancer. J Immunol 167: 2972-2978, 2001.

29. Chen ML, Pittet MJ, Gorelik L, Flavell RA, Weissleder R, von Boehmer $\mathrm{H}$ and Khazaie K: Regulatory T cells suppress tumor-specific CD8 T cell cytotoxicity through TGF-beta signals in vivo. Proc Natl Acad Sci USA 102: 419-424, 2005.

30. Sathaliyawala T, Kubota M, Yudanin N, Turner D, Camp P, Thome JJ, Bickham KL, Lerner H, Goldstein M, Sykes M, Kato T and Farber DL: Distribution and compartmentalization of human circulating and tissue-resident memory $\mathrm{T}$ cell subsets. Immunity 38: 187-197, 2013.

31. Davol PA, Smith JA, Kouttab N, Elfenbein GJ and Lum LG: Anti-CD3 $\mathrm{x}$ anti-HER2 bispecific antibody effectively redirects armed $\mathrm{T}$ cells to inhibit tumor development and growth in hormone-refractory prostate cancer-bearing severe combined immunodeficient beige mice. Clin Prostate Cancer 3: 112-121,2004.

32. Karamouzis MV, Konstantinopoulos PA and Papavassiliou AG: Trastuzumab-mechanism of action and use. N Engl J Med 357: $1664,2007$.

33. Zitron IM, Thakur A, Norkina O, Barger GR, Lum LG and Mittal S: Targeting and killing of glioblastoma with activated $\mathrm{T}$ cells armed with bispecific antibodies. BMC Cancer 13: 83, 2013

34. Baban T, Blomberg C, Hoffner E and Yan X: Anti-HER 2 cancer therapy and cardiotoxicity. Curr Pharm Des: June 4, 2014 (Epub ahead of print).

35. Wang L, He Y, Zhang G, Ma J, Liu C, He W, Wang W, Han H, Boruah BM and Gao B: Retargeting T cells for HER2-positive tumor killing by a bispecific Fv-Fc antibody. PLoS One 8: e75589, 2013 